\title{
Internal conversion coefficients - How good are they now?
}

\author{
T. Kibédi ${ }^{1, a}$, T.W. Burrows ${ }^{2}$, M.B. Trzhaskovskaya ${ }^{3}$, C.W. Nestor, Jr. ${ }^{4}$, and P.M. Davidson ${ }^{1}$ \\ 1 Department of Nuclear Physics, R.S.Phy.S.E., The Australian National University, Canberra, ACT 0200, Australia \\ 2 National Nuclear Data Center, Brookhaven National Laboratory, Upton, NY 11973-5000, USA \\ 3 Petersburg Nuclear Physics Institute, 188300 Gatchina, Russia \\ ${ }^{4}$ University of Tennessee, Knoxville, TN 37996, USA
}

\begin{abstract}
Internal conversion coefficients involving atomic electrons (ICC) and electron-positron pairs (IPC) are often required to determine transition multipolarities and total transition rates. A new internal conversion coefficient data base, BrIcc has been developed which integrates a number of tabulations on ICC and IPC, as well as $\Omega(E 0)$ electronic factors. To decide which theoretical internal conversion coefficient table to use the accurately determined experimental $\alpha_{K}, \alpha_{L}, \alpha_{\text {Total }}$ and $\alpha_{K} / \alpha_{L}$ values were compared with the new Dirac-Fock calculations using extreme assumptions on the effect of the atomic vacancy. While the overall difference between experiment and theory is less than $1 \%$, our analysis shows preference towards the so called Frozen Orbital approximation, which takes into account the effect of the atomic vacancy.
\end{abstract}

\section{Introduction}

Internal conversion coefficients (ICC) convey important information about the atomic nucleus. Through comparison of experimental ICCs with corresponding theoretical values, multipolarities and mixing ratios of nuclear transitions are determined. As well as nuclear structure research, knowledge of accurate coefficients is needed in, for example, the determination of total transition rates (required for the normalization of decay schemes), Mössbauer spectroscopy (CEMS) [1], or nuclear reaction calculations [2].

There is a long history of generation and improvements of theoretical internal conversion coefficient tables. The most recent calculations, based on the relativistic self-consistent Dirac-Fock (DF) method $[3,4]$ represent a major advance in the improvement of the accuracy of the theoretical coefficients, which now challenges experiments at the percent level. To make the new theoretical values accessible for a very broad user community a new internal conversion coefficient data base called BrIcc has been developed [5], which is now adopted by the International Nuclear Structure and Decay Data Network [6] for all new data evaluations published in Nuclear Data Sheets.

To assess the question of How good are the theoretical internal conversion coefficients? accurately measured values were used from time to time to carry out benchmark studies; see for example the work of Raman et al. [4]. This paper is focused on the critical evaluation of existing internal conversion coefficient data using rigorous statistical methods to compare adopted values with the DF values.

\section{High precision experimental internal conversion coefficients}

In the simplest way the ICC is defined as $\alpha=I_{C E} / I_{\gamma}$, where $I_{C E}$ and $I_{\gamma}$ are the electron and $\gamma$-ray intensities, respectively.

${ }^{a}$ Presenting author, e-mail: Tibor.Kibedi@anu.edu.au
$I_{C E}$ represents the number of electrons ejected from an atomic shell (K, L1, L2...), a major shell (L, M, etc.), or the sum of all shells (total), or can be the intensity of the electronpositron pairs. The internal conversion coefficient depends on the atomic number of the emitting nucleus $(Z)$, the transition energy $\left(E_{\gamma}\right)$, the transition multipolarity $(\pi L)$ and, in the case of internal conversion, the atomic shell involved. Often the ratio of different ICCs, for example $\alpha_{K} / \alpha_{L}$ or $\alpha_{L 1} / \alpha_{L 2}$, was used to determine the multipolarity and/or the mixing ratio. Internal conversion coefficients also can be deduced from the intensity of X-rays or Auger electrons, which are emitted as the atomic vacancies are filled.

The measurement of internal conversion coefficients, including the methods, instrumentation and data analysis, has been developed considerably over the last 60 years, as is illustrated in figure 1, which shows the evolution of the experimental and theoretical K-shell internal conversion coefficient for the $661.657 \mathrm{keV}$ M4 transition in ${ }^{137} \mathrm{Ba}$. This is one of the most studied ICC; there are 29 experimental values known. In assembling the ICC data base individual data points with less than $15 \%$ experimental uncertainties have been considered; however, in the further analysis comparing experiment with theory the adopted ICC must be known to better than $5 \%$ accuracy.

We only considered $\alpha_{K}, \alpha_{L}$ and $\alpha_{T}$ internal conversion coefficients and $\alpha_{K} / \alpha_{L}$ ratios with $\mathrm{L} \geq 2$ (E2, E3, M3, E4, M4 and E5) multipolarity. Electric dipole transitions are usually hindered strongly. Transitions of E1, M1 and M2 multipolarities are often mixed and the mixing ratios not always known to the accuracy needed to define the ICC values. For M1 and M2 transitions the nuclear penetration effect also could have an impact on the measured internal conversion coefficients. The determination of the multipolarities has been examined and those transitions whose multipolarity assignment is solely based on the particular ICC value were not considered. Similarly, if an ICC was used for normalization it was excluded. The transition energy and uncertainty, the multipolarity and the mixing ratio with its uncertainty (mixed 


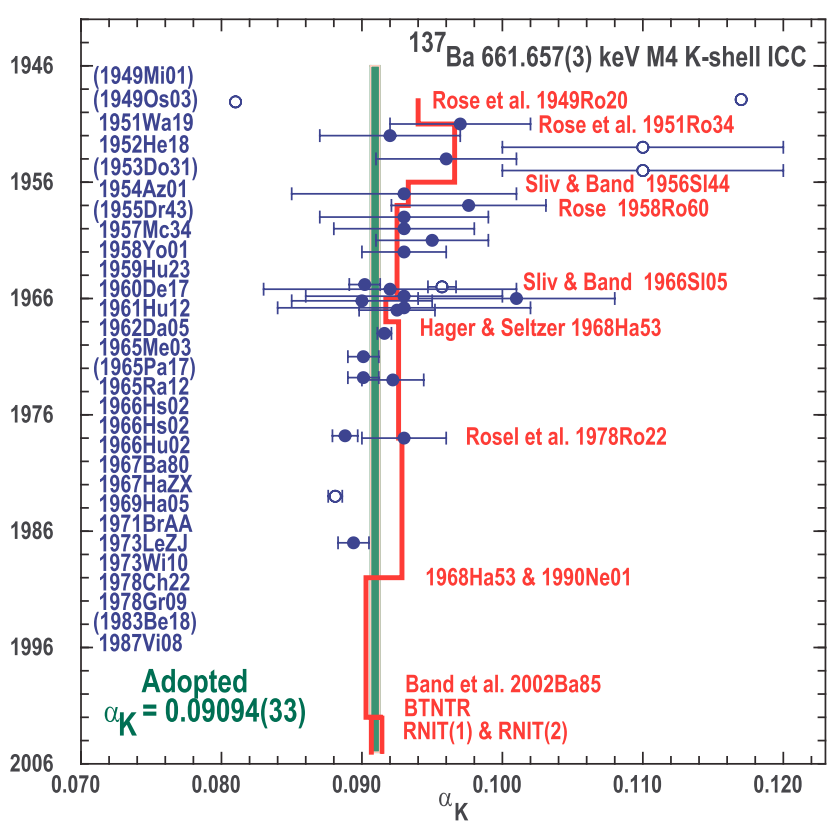

Fig. 1. Adopted value (shaded area) of the experimental $\alpha_{K}$ for the $662 \mathrm{keV}$ M4 transition in ${ }^{137} \mathrm{Ba}$. Data points: filled included in the present evaluation; open excluded. Lines (red in on-line) show the evolution of the corresponding $\alpha_{\text {Theo }}$ values. References (experiments on the left; theory on the right) are given as NSR [7] keynumbers.

transitions only) were taken from the adopted data set of the ENSDF [8].

There are a number of techniques developed to measure internal conversion coefficients [9], which usually require the knowledge of the intensity of two radiations, including conversion electrons, $\gamma$-rays, X-rays, $\beta$-rays, etc. In our review no preference was given to any of the methods, but in order to deduce the correct ICC significant effort went to examine every piece of information and to check if the best data were used for calibration, normalization, etc. For example the so called XPG method is based on the fact that each electron vacancy created in the $\mathrm{K}$ shell produces $\mathrm{K}$ X-Rays with a probability equal to the K-shell fluorescent yield $\omega_{K}$. The value of $\omega_{K}$ depends only on the atomic number, and is known with an accuracy of $\sim 0.5 \%$ [10]; however, its value has changed quite significantly over the last decades. For many of the E2 transitions the total internal conversion coefficients, $\alpha_{T}$ of have been deduced by combining the reduced E2 electromagnetic transition probability, B(E2), obtained from Coulomb excitation with the half-life of the level, $T_{1 / 2}$, from lifetime measurements. The basic formula of the so-called CEL method is defined by Raman et al. [4] as:

$$
T_{1 / 2}(n s) \times\left(1+\alpha_{T}\right)=\frac{2.829 \times 10^{11} \times E_{\gamma}^{-5}(\mathrm{keV})}{B(E 2) \uparrow\left(e^{2} b^{2}\right)} .
$$

Special consideration was given in deriving the uncertainties of $\alpha_{T}$. Assuming a Gaussian distribution for both $T_{1 / 2}$ and $B(E 2), \Delta \alpha_{T}$ may not be Gaussian due to the inverse relationship with $T_{1 / 2}$ and $B(E 2)$. We have adopted a procedure to estimate the uncertainty correctly taking into account this nonlinear relation.
In summary, in about $22 \%$ of the cases, the ICCs (or uncertainties) have been adjusted from values originally reported. When more than one experimental value was known, adopted values were deduced using procedures described below.

There is an overlap of our data base and the two recent compilations of experimental values by Raman et al. [4] in 2002 and [13] in 2006. The $100 \alpha_{K}$ and $\alpha_{T}$ values of [4] have been selected on similar principles to ours; however, a large number of corrections have been made in the verification procedures presented here. More recently [13] an extensive set of 1510 internal conversion coefficient ratios, measured with an accuracy of $\leq 10 \%$ has been compiled, but no attempt was made to deduce adopted values.

\section{Comparing experimental and theoretical ICCs}

In comparing the experimental ICC values with a theoretical ones we have adopted the definition of [4] for the difference of $\Delta_{\text {ICC }}($ Exp: Theor), given in percentage as:

$$
\Delta_{\text {ICC }}(\operatorname{Exp}: \text { Theor })=\frac{\alpha_{i}(\text { Exp })-\alpha_{i}(\text { Theor })}{\alpha_{i}(\text { Theor })} \times 100,
$$

where $i$ refers to a particular ICC value of Total, K-shell, $\mathrm{L}$-shell or $\mathrm{K} / \mathrm{L}$ ratio, etc. Theoretical internal conversion coefficients, $\alpha_{i}$ (Theor) were calculated using the original RAINE program [3] in combination with BrIcc [6]. This approach eliminates a small, $\leq 0.3 \%$, systematic uncertainty of the interpolation procedure. The uncertainty of the $\alpha_{i}$ (Theor) values has been determined from the uncertainty in transition energy and, in the case of mixed multipolarity transitions, from the uncertainty of the the mixing ratio [6].

One remaining central question concerning the theoretical ICC calculations is the treatment of atomic vacancies created during the conversion process. The new Dirac-Fock model of Band et al. [3] offers two extreme assumptions to take into account the effect of the atomic vacancy:

- The hole is filled instantaneously, therefore the conversion process is not affected; BTNTR or No Hole approximation.

- The hole remains unfilled throughout the time that the conversion electron is present in the atom; RNIT(1) or Self Consistent approximation. An alternative approximation, RNIT(2) or Frozen Orbital, uses a somewhat different potential in calculating the continuum wave function.

In the lack of sufficient theoretical arguments the decision to select one of the theoretical approximations must be based on careful comparison of the measured and the calculated ICCs. Figure 2 compares the values of the total ICCs calculated with the No Hole and with the Frozen Orbital approximations and for $\mathrm{E} 2$ and M4 transitions in $Z=20,50$ and 80 . The frequency of all known $\alpha_{\text {Total }}$ values in the ENSDF is also shown in the figure, which can be used as a guide to illustrate the impact of the selection of the ICC data table for future evaluations.

\section{Statistical treatment of the data}

Three statistical techniques [11] were employed to analyze the data aiming to identify discrepant data points and deduce 


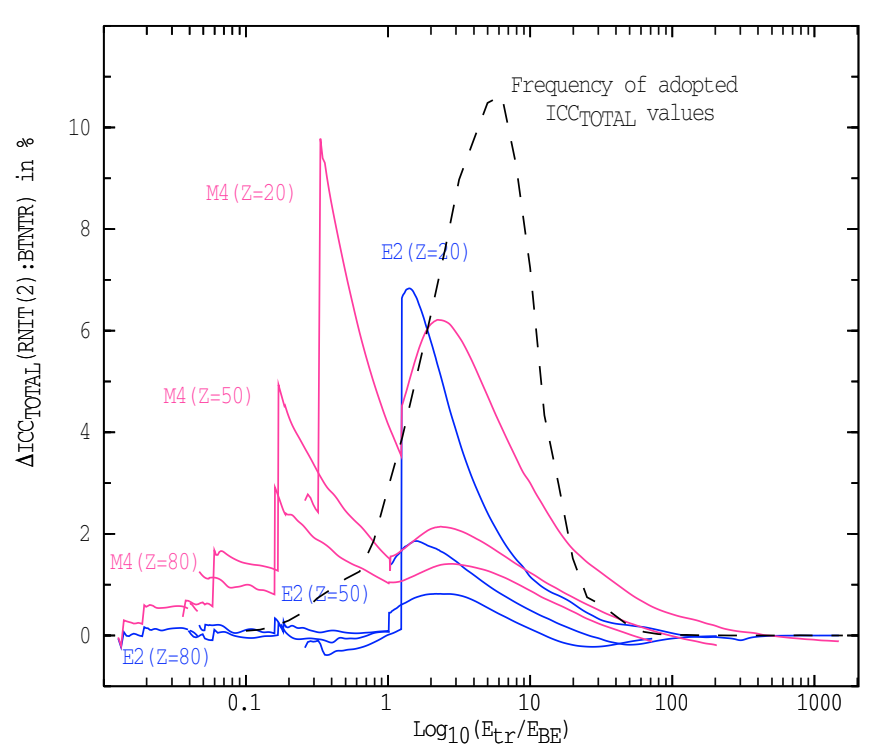

Fig. 2. Differences (in \%) of the $\alpha_{T}$ values calculated using the No Hole (BTNTR) and Frozen Orbital (RNIT(2)) approximations. The horizontal axis is the transition energy $\left(E_{t r}\right)$ divided by the K-shell binding energy $\left(E_{B E}\right)$. Dashed curve shows (in \%) the frequency spectrum of the known $\alpha_{T}$ values in the adopted ENSDF data sets [8].

weighted mean values and assign uncertainties. These methods were the

- Limitation of Relative Statistical Weights Method (LWM),

- Normalized Residuals Method (NRM), and

- Rajeval Technique (RT).

If a set of data is consistent, these three methods will yield the same mean value and uncertainty and, in the case of LWM and $\mathrm{NRM}$, the same reduced $\chi^{2}$. If the data set is discrepant, the results of these techniques will differ since they use different methods in treating discrepant data. These differences were used to identify deviant data points. Indications of a deviant data point could include that it was marked as outlier by LWM or RT; or its uncertainty was adjusted (increased) by RT or NRM. The procedure was repeated until no more deviant data point could be identified. Somewhat different procedures were used in the analysis of the experimental data and in the comparison of experiment to calculations. In the first case the adopted experimental value was taken as the arithmetic mean of the NRM and RT and the uncertainty was the larger of the NRM and RT uncertainties (providing that more than two measurements were included in the calculation). In the comparison of experiments to theory the mean value, uncertainty and reduced $\chi^{2}$ of the $\Delta_{I C C}$ (Exp: Theor) differences were taken from LWM.

\section{Summary}

A total of 213 experimental ICC has been adopted, which are known to better than 5\% accuracy. The data set covers the $\mathrm{Z}=20\left({ }^{44} \mathrm{Ca}\right)$ to the $\mathrm{Z}=94\left({ }^{240} \mathrm{Pu}\right)$ atomic numbers and the $24 \mathrm{keV}\left({ }^{58} \mathrm{Co}\right)$ to $1238 \mathrm{keV}\left({ }^{56} \mathrm{Fe}\right)$ energy range. The total data set has been analyzed in various subgroups and compared with three different theoretical approximations,
Table 1. Average differences (in \%) between accurately measured and theoretical internal conversion coefficients.

\begin{tabular}{ccclcc}
\hline $\begin{array}{c}\text { Multi- } \\
\text { polarity }\end{array}$ & Shell & $\mathrm{N}$ & \multicolumn{3}{c}{$\bar{\Delta}_{\text {ICC }}$ (Exp:Theor) } \\
\hline & & & BTNTR & RNIT(1) & RNIT(2) \\
All & All & 186 & $+0.70(40)^{a)}$ & $-0.61(14)$ & $-0.93(14)$ \\
All & $\mathrm{T}$ & 54 & $+0.32(25)$ & $-0.55(24)$ & $-0.71(24)$ \\
All & $\mathrm{K}$ & 72 & $+1.50(120)^{a)}$ & $-0.18(21)$ & $-0.72(21)$ \\
All & $\mathrm{L}$ & 9 & $+0.33(76)$ & $+0.06(76)$ & $-0.06(76)$ \\
All & K/L & 46 & $+0.00(31)$ & $-1.64(31)$ & $-1.94(30)$ \\
All & T\&K & 126 & $+0.99(69)^{a)}$ & $-0.34(16)$ & $-0.71(16)$ \\
& & & & & \\
E2 & All & 103 & $+0.21(23)$ & $-0.77(23)$ & $-0.93(23)$ \\
E2 & T & 36 & $-0.07(41)$ & $-0.70(41)$ & $-0.81(41)$ \\
E2 & $\mathrm{K}$ & 35 & $+1.06(43)$ & $-0.04(43)$ & $-0.25(43)$ \\
E2 & K/L & 23 & $-0.08(42)$ & $-1.68(43)$ & $-2.12(43)$ \\
& & & & & \\
E3 & All & 18 & $+0.83(39)$ & $-0.69(37)$ & $-1.07(37)$ \\
& & & & & \\
M4 & All & 50 & $\left.+0.98(68)^{a}\right)$ & $-0.51(20)$ & $-0.93(20)$ \\
M4 & T & 12 & $+0.40(36)$ & $-0.65(36)$ & $-0.82(36)$ \\
M4 & K & 20 & $+1.81(151)^{a}$ & $+0.00(28)$ & $-0.69(28)$
\end{tabular}

High precision ICCs $(\Delta \alpha / \alpha \leq 1.5 \%)$

All All $\quad 25 \quad+0.77(51) \quad-0.56(26) \quad-0.95(17)$

${ }^{a}$ LWM has expanded the uncertainty so the range includes the most precise input value.

as listed in table 1 . If a $\Delta_{I C C}($ Exp: Theor) difference for a particular experimental ICC was found to be discrepant for all three theoretical calculations, the data point was excluded from the analysis. This procedure has reduced the total number of experimental ICC values to 186. Figures 3 and 4 show in detail 72 accurately determined $\alpha_{K}(\operatorname{Exp})$ internal conversion coefficients compared with the No Hole and the Frozen Orbital approximations. The horizontal axis is the $E_{t r} / E_{B E}$ ratio, which transforms the energy dependence of the $\Delta_{I C C}($ Exp: Theor) differences to the same scale irrespective of the atomic number. A notable feature of figure 3 is the correlation between the $E_{t r} / E_{B E} \leq 3$ data points. As is shown in figure 2, large differences between calculated ICCs are expected close to the shell binding energy. One of the important

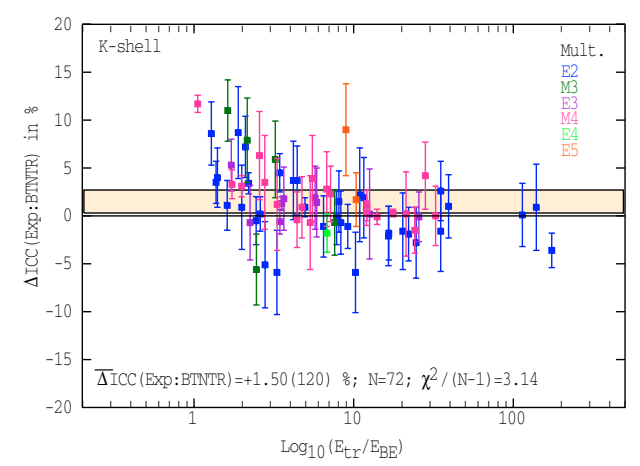

Fig. 3. Adopted value (shaded area) of the average difference between experimental $\alpha_{K}$ values and calculated ones using the so called No Hole, BTNTR approximation. 


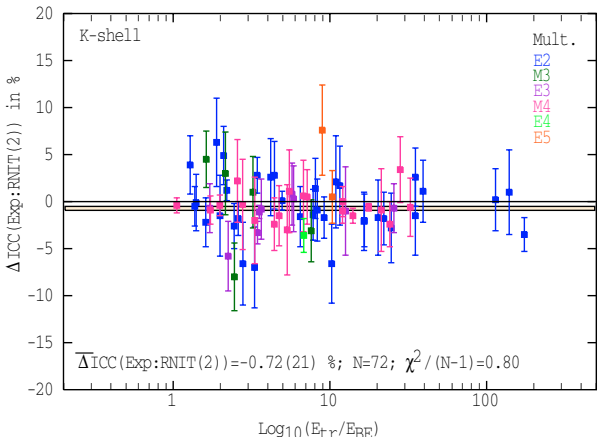

Fig. 4. Same as figure 3 but for the Frozen Orbital, RNIT(2) approximation.

data points shown is the $80.2369(7) \mathrm{keV}$ pure M4 transition in ${ }^{193} \mathrm{Ir}$, which is only $4.126 \mathrm{keV}$ above the $\mathrm{K}$-shell binding energy of $76.111 \mathrm{keV}$. The most accurate experimental value of $\alpha_{K}(\operatorname{Exp})=103.0(8)$ reported recently by Nica et al. [12] is more than $10 \%$ higher than the $\alpha_{K}(B T N T R)=92.18(12)$ obtained with the No Hole approximation. When the adopted experimental $\alpha_{K}$ data set is compared with the theoretical values calculated by the Self Consistent and the Frozen Orbital approximation slightly smaller average $\Delta_{I C C}($ Exp:Theor) differences were obtained. For the $80.2369 \mathrm{keV}$ M4 transition the $\alpha_{K}(R N I T(1))=99.63(12), \Delta_{I C C}(\operatorname{Exp}: R N I T(1))=+3.4(8) \%$ and $\alpha_{K}(R N I T(2))=103.45(12), \Delta_{I C C}(\operatorname{Exp}: R N I T(2))=$ $-0.4(8) \%$. The ratio of $\mathrm{K}$-shell internal conversion coefficients for the $127.5-\mathrm{keV} \mathrm{E} 3$ transition in ${ }^{134} \mathrm{Cs}$ and the $661.7-\mathrm{keV} \mathrm{M} 4$ transition in ${ }^{137} \mathrm{Ba}$ determined accurately by Nica et al. [14] also prefers the Frozen Orbital approximation and disagrees by more than three standard deviations with the No Hole calculation.

The main aim of our review was to assemble an experimental data base and to compare it to the various theoretical calculations. The average difference in table 1 between all experimental data and the new Dirac-Fock model [3] is smaller than $1 \%$; however, there are some differences in terms of atomic shell and transition multipolarity. It is also evident that the No Hole (BTNTR) approximation tends to underestimate experiments, but in some cases $\bar{\Delta}_{I C C}(\operatorname{Exp}: B T N T R)$ values show large fluctuations; for example for the $\alpha_{K}$ and the $\alpha_{\text {Total }}$ values for "All" (see table 1) and E2 multipolarities. On the other hand the two approximations, Self Consistent (RNIT(1)) and Frozen Orbital (RNIT(2)) tend to overestimate experiment. However the average differences between the various subgroups in table 1 do not fluctuate that much. Owing to the fact that almost all transition energies considered were higher than the K-shell binding energy for the decaying atom, sizable differences in the theoretical ICC values are only expected for the K-shell. It should be noted that the calculated ICC values close to the binding energy of $\mathrm{L}, \mathrm{M}$, etc. atomic shells are also sensitive to the choice of the physical model.
As it is shown in the last entry of table 1,25 experimental ICC out of the 186 included in our analysis are known to better than $1.5 \%$ relative uncertainty. The weighted mean values of the $\bar{\Delta}_{I C C}$ (Exp:Theor) differences of this group, comprising 10 $\alpha_{K}, 1 \alpha_{L}, 5 \alpha_{K / L}$ and $9 \alpha_{T o t}$ data, are very similar to the corresponding values obtained for the total data set; however, the uncertainty is factor three larger for No Hole (BTNTR) than for Frozen Orbital (RNIT(2)). The reduced $\chi^{2}$ values of BTNTR: 8.2, RNIT(1): 2.12 and RNIT(2): 1.06 (for $\mathrm{N}=$ 25 the critical value at $99 \%$ confidence level is 1.79 ). This provides a further evidence to support our preference toward the Frozen Orbital (RNIT(2)) approximation.

There are other atomic or higher order effects which could influence the ICC values. For example, atomic many body correlations, a non-spherical atomic field in the case of partially filled shells, the uncertainty in the binding energy, the chemical environment of the atom, etc. It is assumed that these effects are important close to the shell binding energy. To minimize their impact BrIcc uses an ICC data table starting $1 \mathrm{keV}$ above the shell binding energy.

\section{References}

1. A. Blachowski, K. Ruebenbauer, Phys. Scr. 73, 263 (2006).

2. T. Belgya et al., RIPL-2, IAEA-TECDOC-1506 (IAEA, Vienna, 2006).

3. I.M. Band, M.B. Trzhaskovskaya, C.W. Nestor Jr., P.O. Tikkanen, S. Raman, At. Data Nucl. Data Tables 81, 1 (2002).

4. S. Raman, C.W. Nestor Jr., A. Ichihara, M.B. Trzhaskovskaya, Phys. Rev. C 66, 044312 (2002).

5. T. Kibédi, T.W. Burrows, M.B. Trzhaskovskaya, C.W. Nestor Jr., Proc. Int. Conf. Nuclear Data for Science and Technology, Santa Fe, New Mexico, 2004, Vol. 1, edited by R.C. Haight, M.B. Chadwick, T. Kawano, P. Talou, p. 268; AIP Conf. Proc. 769 (2005).

6. T. Kibédi, T.W. Burrows, M.B. Trzhaskovskaya, C.W. Nestor Jr., ANU-P/1684 (2005); Program can be obtained from: http://www.nndc.bnl.gov/nndcscr/ensdf_pgm/analysis/BrIcc/; A web interface is at: www.rsphysse.anu.edu.au/nuclear/bricc/.

7. Nuclear Science References, http://www.nndc.bnl.gov/nsr/.

8. Evaluated Nuclear Structure Data File, http://www.nndc.bnl.gov/ensdf/.

9. J.H. Hamilton, in The Electromagnetic Interaction in Nuclear Spectroscopy (North-Holland, 1975), p. 441.

10. E. Schonfeld, H. Janssen, Nucl. Instrum. Meth. Phys. Res. A 369, 527 (1996).

11. M.U. Rajput, T.D. Mac Mahon, Nucl. Instrum. Meth. Phys. Res. A 312, 289 (1992).

12. N. Nica, J.C. Hardy, V.E. Iacob, S. Raman, C.W. Nestor Jr., M.B. Trzhaskovskaya, Phys. Rev. C 70, 054305 (2004).

13. S. Raman, M. Ertugrul, C.W. Nestor Jr., M.B. Trzhaskovskaya, At. Data Nucl. Data Tables 92, 207 (2006).

14. N. Nica, J.C. Hardy, V.E. Iacob, W.E. Rockwell, M.B. Trzhaskovskaya, Phys. Rev. C 75, 024308 (2007). 